\title{
Thermoelectric Properties of Polymeric Mixed Conductors
}

Ujwala Ail, Mohammad J avad J afari, Hui Wang, Thomas Ederth, Magnus Berggren and Xavier Crispin

The self-archived postprint version of this journal article is available at Linköping University Institutional Repository (DiVA):

http:// urn.kb.se/ resolve?urn=urn:nbn:se:liu:diva-133171

N.B.: When citing this work, cite the original publication.

Ail, U., J afari, M. J ., Wang, H., Ederth, T., Berggren, M., Crispin, X., (2016), Thermoelectric Properties of Polymeric Mixed Conductors, Advanced Functional Materials, 26(34), 6288-6296.

https:// doi.org/ 10.1002/adfm.201601106

Original publication available at:

https:// doi.org/ 10.1002/adfm.201601106

Copyright: Wiley (12 months)

http:// eu.wiley.com/WileyCDA/ 


\title{
Thermoelectric Properties of Polymeric Mixed Conductors
}

Ujwala Ail ${ }^{1}$, Mohammad Javad Jafari ${ }^{2}$, Hui Wang ${ }^{1}$, Thomas Ederth ${ }^{2}$, Magnus Berggren ${ }^{1}$, Xavier $\operatorname{Crispin}^{1 *}$

1. Department of Science and Technology, Linköpings Universitet, S-60174 Norrköping, Sweden

2. Department of Physics, Chemistry and Biology, Linköping University, S-581 83 Linköping

Sweden

*E-mail: xavier.crispin@liu.se

Keywords: mixed ion-electron conductors, ionic Seebeck effect, mixed conducting polymers, poly(3,4-ethyelenedioxythiophene)-polystyrene sulfonate, ATR-FTIR spectroscopy

\begin{abstract}
The thermoelectric (TE) phenomena are intensively explored by the scientific community due to the rather inefficient way energy resources are used with a large fraction of energy wasted in the form of heat. Among various materials, mixed ion-electron conductors (MIEC) are recently being explored as potential thermoelectrics, primarily due to their low thermal conductivity. The combination of electronic and ionic charge carriers in those inorganic or organic materials leads to complex evolution of the thermovoltage $\left(\mathrm{V}_{\text {oc }}\right)$ with time, temperature and/or humidity. One of the most promising organic thermoelectric materials, poly(3,4-ethyelenedioxythiophene)-polystyrene sulfonate (PEDOT-PSS), is a MIEC. A previous study reveals that at high humidity, PEDOT-PSS undergoes an ionic Seebeck effect due to mobile protons. Yet, this phenomenon is not well understood. In this work, we study the time dependence of the $\mathrm{V}_{\text {ос }}$ and explain its behavior from the contribution of both charge carriers (holes and protons). We identify the presence of a complex reorganization of the charge carriers promoting an internal electrochemical reaction within the polymer film. Interestingly, we demonstrate that the time dependence behavior of $\mathrm{V}_{\text {oc }}$ is a way to distinguish between three classes of polymeric materials: electronic conductor, ionic conductor and mixed ionic- electronic conductor.
\end{abstract}




\section{Introduction}

Mixed ionic-electronic conductors (MIEC) are widely used in devices for energy conversion and storage. ${ }^{[1-8]}$ MIECs are materials that conduct both ions and electrons. ${ }^{[9]}$ They include semiconductors and solid electrolytes. Besides the standard electronic and ionic conductors, MIEC s belong to two distinct families, namely, ceramics and conducting polymers. Survey of the state-ofthe- art in solid state electronic, ionic and mixed conductors has been summarized by Malti et al. ${ }^{\text {[10] }}$ It is important to note that there are limited number of MIECs with both high electronic and ionic conductivities. Since single phase MIECs rarely fulfill these requirements, composite materials consisting of separate ionic and electronic conductive phases are often adapted as a solution to this challenge. ${ }^{[11]}$ There is a lot of interests in MIECs with respect to their thermoelectric properties. Apart from $\mathrm{Zn}_{4} \mathrm{Sb}_{3}$, there has been extensive studies on super-ionic conductors; $\mathrm{Cu}_{2-x} \mathrm{Se}$, $\mathrm{Cu}_{1.97} \mathrm{Ag}_{0.03} \mathrm{Se}, \mathrm{Cu}_{1.97} \mathrm{Ag}_{0.03} \mathrm{Se}_{1+\mathrm{y}}, \mathrm{Ag}_{2+\mathrm{x}} \mathrm{Se}, \mathrm{AgCrSe}_{2}$, etc. ${ }^{[12-20]}$ as they undergo a structural phase transition above which the metal ions become mobile leading to enhanced phonon scattering, consequently leading to low thermal conductivity. They also have remarkable electrical conductivity. In case of $\mathrm{Cu}_{2} \mathrm{Se}$ and $\mathrm{Cu}_{1.97} \mathrm{Ag}_{0.03} \mathrm{Se}$ (holes are the majority carriers except at the highest temperatures) thermopower or Seebeck coefficient, $\alpha$ (where, $\alpha=\frac{V_{o c}}{\Delta T}, \Delta \mathrm{T}$ is the temperature difference) versus temperature, shows dramatic peak (55\% increase in $\alpha$ ) around the superionic phase transition temperature (characterized by disordered $\mathrm{Cu}^{+}$above the phase transition) with increased phonon and electron scattering. ${ }^{[13]}$ It has been suggested that a great portion of the $\alpha$ increase might be explained by the coupling of structural degrees of freedom associated with the phase transition to carrier transport, indicating the use of structural entropy for enhanced $\alpha$ as new approach for enhancing thermoelectric figure of merit, ZT (where, ZT= $\sigma \alpha^{2} \mathrm{~T} / \lambda$, where $\sigma, \lambda$ and T are electrical conductivity, thermal conductivity and the absolute temperature respectively). Korzhuev et al. ${ }^{\text {[21] }}$ measured a sharp peak in the thermodiffusion of $\mathrm{Cu}^{0}$ on $\mathrm{Cu}_{2} \mathrm{Se}$ at the phase transition and the corresponding $\alpha$ was calculated to be $\alpha_{\mathrm{Cu}^{+}}-\alpha_{p}=2500 \mu \mathrm{V} / \mathrm{K}$, implying a very large value for $\alpha_{\mathrm{Cu}^{+}}$at the phase transition. A significant change in $\alpha$ (up to $400 \mu \mathrm{V} / \mathrm{K}$ ) is also observed in $\mathrm{Ce}_{1-\mathrm{x}} \mathrm{Gd}_{\mathrm{x}} \mathrm{O}_{2-\mathrm{x} / 2-\delta}$ 
as a function of oxygen partial pressure in the transition region from electronic to ionic conduction. [22]

Conducting polymers based on poly(3,4-ethylene- dioxythiophene); PEDOT are appealing in this regard as they are known to exhibit both electronic and ionic conduction. Owning to this property, they have been investigated extensively for their reversible electrochemical activity.

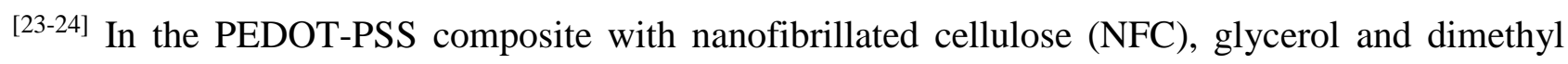
sulfoxide (NFC-PEDOT paper) Malti et al. reported high electronic and ionic conductivity. ${ }^{\text {[10] }}$ PEDOT with Tosylate (Tos) or PSS counter ions display attractive thermoelectric properties with the highest reported values for the power factor,$\sigma \alpha^{2}$ of $1270 \mathrm{~W} \mathrm{~m}^{-1} \mathrm{~K}^{-2},{ }^{[25]}$ and ZT of $0.25,{ }^{[26]} 0.31$, ${ }^{[27]}$ and $0.42,{ }^{[28]}$ at room temperature. Interestingly, the humidity was also found to be a crucial factor, increasing the $\sigma \alpha^{2}$ from 23 to $355 \mu \mathrm{Wm}^{-1} \mathrm{~K}^{-2}$. ${ }^{[29]}$ This rather large increase in $\sigma \alpha^{2}$ was attributed to the morphological changes due to the absorption of water and/or electrochemical reactions of PEDOT upon exposure to air. Recently, we reported the role of ions in the thermoelectric response of different PEDOT derivatives. A large resulting thermopower, reaching several hundreds of $\mu \mathrm{V} / \mathrm{K}$ at high humidity levels, was identified to be due to primarily ionic Seebeck effects. ${ }^{[30]}$ However, the high $\mathrm{V}_{\text {oc }}$ observed in mixed electron-ion conductors, such as for PEDOT-PSS, cannot be sustained, but decreases with time due to the compensation and polarization of ions and electronic charges that occur in such systems.

In this study, we aim at understanding the underlying mechanism of the timedependence of the $\mathrm{V}_{\text {ос }}$ in a mixed ionic-electronic conductor such as for PEDOT-PSS. By comparing the thermoelectric characteristics between a pure electronic conductor (PEDOT-Tos), a pure ionic conductor (PSS) and a mixed electron-ion conductor (PEDOT-PSS), we identify, for the first time, that an internal electrochemical reaction is induced within the conducting polymer layer promoted by the ionic thermoelectric effect. 


\section{Results}

\subsection{Electric and thermoelectric characterization}

In order to understand the thermoelectric properties of the MIEC, PEDOT-PSS under different humidity levels, we systematically compare its properties with those of PEDOT-Tos (primarily an electronic conductor) and the polyelectrolyte, poly(sodium-4-styrene sulfonate), PSSNa (solely an ion conductor). Figure 1 shows the structures and the kind of electrical charge carriers present in the three different polymer systems. Figure 2a displays the electrical conductivity obtained from impedance spectroscopy as a function of the relative humidity $(\mathrm{RH})$ for PEDOT-Tos, PEDOTPSS and PSSNa. PEDOT-Tos (a thickness of $627 \mathrm{~nm}, \sigma=15200 \mathrm{Sm}^{-1}$ at RT and 10\%RH) was prepared by in situ oxidative polymerization process. ${ }^{[31]}$ PEDOT-PSS films (a thickness of $5.68 \mu \mathrm{m}$, $\sigma=14 \mathrm{Sm}^{-1}$ at $\mathrm{RT}$ and $10 \% \mathrm{RH}$ ) were manufactured from a commercial water dispersion (Baytron P), provided by H. C. Starck (1.3 wt\% PEDOT-PSS). PSSNa ( Mw: 70,000g/mol) was purchased from Sigma-Aldrich and films (thickness $1.5 \mu \mathrm{m}, \sigma=9.58 \times 10^{-4} \mathrm{Sm}^{-1}$ at $\mathrm{RT}$ and $10 \% \mathrm{RH}$ ) were obtained by drop-casting the solution onto the planar glass substrates ( $2 \mathrm{wt} \%$ PSSNa in deionized water). The total electrical conductivity in the polymer films is the sum of the electrical and ionic conductivity components. Humidity is known to facilitate and also modulate the ionic conductivity in polymers, and therefore the study of the conductivity versus a change in the RH gives us an insight regarding the relative levels of the electronic versus the ionic conductivity. As can be seen in Figure 2a, the conductivity of PEDOT-Tos is almost independent versus the RH. The most predominant conduction is then represented by electronic charges, since the large size of the tosylate counter ion do not promote fast ion conduction even at high RH levels. ${ }^{[31]}$ In the case of the polyelectrolyte, the PSSNa system, the sulfonate groups are dissociated from the sodium cation. The presence of water then aids in screening the electrostatic attraction of the cations with the immobile sulfonate groups of the polyanion; thus resulting in an increase in mobility of the sodium cations. ${ }^{[32]}$ The overall 
conductivity in the PSSNa system exhibits an exponential increase with the $\mathrm{RH}$, indicating that ionic conduction represents the electrical conductivity in this material. ${ }^{[33]}$ PEDOT-PSS is known as a MIEC, wherein both ionic (cations) and the electronic (holes) charge carriers contribute to the overall electrical conduction. PEDOT-PSS film exhibits a decrease in the electrical conductivity up to $40 \%$ $\mathrm{RH}$, while above this level the conductivity increases with RH. Even though the ionic conductivity increases by one order of magnitude with humidity, the electrical transport is still dominated by electronic conduction. The details of the composition of the electrical conductivity behavior for PEDOT-Tos and PEDOT-PSS vs. humidity have been reported in a previous communication. ${ }^{[30]}$

The set-up used for the $\mathrm{V}_{\text {ос }}$ measurement is depicted in Figure $\mathbf{2 b}$ and further explained in the experimental part. Figure 2c shows the evolution of the open circuit voltage $\left(\mathrm{V}_{\text {oc }}\right)$ with time for the three samples, as a temperature difference $(\Delta \mathrm{T})$ of $1^{\circ} \mathrm{C}$ is applied between the two electrodes at $80 \% \mathrm{RH}$. In case of PEDOT-Tos, the electronic conductor, $\mathrm{V}_{\text {ос }}$ increases as $\Delta \mathrm{T}$ increases and saturates at a constant value of $9 \mu \mathrm{V}$ within $\sim 30$ s. Hence, the development of the voltage $\left(\mathrm{V}_{\text {oc }}\right)$ follows the temperature rise and it is thus almost instantaneous. For PSSNa, i.e. the ionic conductor, the evolution of $\mathrm{V}_{\text {ос }}$ is completely decoupled with the increase characteristics of $\Delta T$. $V_{\text {oc }}$ reaches a high voltage ranging 5-10 mV after a period of time of about $100 \mathrm{~min}$; the $\Delta \mathrm{T}$ reaches a static value in typically $30 \mathrm{~s}$. The thermodiffusion of ions in polyelectrolytes, due to the Soret effect, is known to lead to large $\mathrm{V}_{\text {ос }}$ in the $\mathrm{mV} / \mathrm{K}$ range. ${ }^{[34]}$ For the mixed conductor PEDOT-PSS, the $\mathrm{V}_{\text {ос }}$ evolves in a complex fashion. $\mathrm{V}_{\text {oc }}$ increases steadily within $30 \mathrm{~s}$, as $\Delta \mathrm{T}$ is increased, reaching a maximum value (dependent on the $\mathrm{RH}$ ), before the $\Delta \mathrm{T}$ stabilization time. Nonetheless, after that $\mathrm{V}_{\text {oc }}$ reaches the maximum value, it then starts to decrease, thus forming a peak. The value then settles to a minimum value of around $4-10 \mu \mathrm{V}$ corresponding to the $\mathrm{V}_{\text {ос }}$ of the electronic charge carriers. The peak value of the $\mathrm{V}_{\text {oc }}$ depends on the ambient humidity and the value reaches as high as $380 \mu \mathrm{V}$ at $100 \% \mathrm{RH}$, see Figure 3a. In comparison to the traditional relatively much smaller electronic contribution (found in PEDOT-Tos), the presence of this high, but short-term lasting, $\mathrm{V}_{\text {ос }}$ suggest a dynamic contribution from an ionic Seebeck effect (as found in PSSNa). Figure 3a shows the influence of the RH on the 
$\mathrm{V}_{\text {oc }}$ evolution when a $\Delta \mathrm{T}$ of $1^{\circ} \mathrm{C}$ is applied across the PEDOT-PSS sample. The inset of Figure 3a displays the $\mathrm{V}_{\text {oc }}$ peak value per $1{ }^{\circ} \mathrm{C}$ for the PEDOT-PSS film at various $\mathrm{RH}$ values ranging from $20 \%$ to $100 \%$. The fact that the $\mathrm{V}_{\text {oc }}$ peak value depends on the humidity level is yet another indication that the origin for this $\mathrm{V}_{\text {ос }}$ versus time evolution includes a contribution from ionic transport.

\subsection{Hypothesis of $V_{\text {oc }}$ Evolution in Mixed Conductors}

When a $\Delta \mathrm{T}$ is applied, it is reasonable to consider the thermodiffusion of holes from the hot side towards the cold side. This leads to an increased concentration of holes at the cold side, resulting a $\mathrm{V}_{\text {oc }}$ in the range of $\sim 20 \mu \mathrm{V}$ in the region (i) of the curve in Figure $3 \mathbf{b}$. Since PEDOT-PSS is a mixed conductor, in addition to holes, it is expected to have significant thermodiffusion of protons towards the cold end, creating an internal electric field that would dominate the one developed by the thermodiffusion of holes (for the sake of comparison of the magnitude of those effects, one can remind the values for the pure electronic and ionic conductors: $\mathrm{S}_{\mathrm{h}}{ }^{+}$in PEDOT-Tos $(\sim 20 \mu \mathrm{V} / \mathrm{K})<$ $\mathrm{S}_{\mathrm{Na}}{ }^{+}$in PSSNa (5-10 mV/K). The ionic contribution is reflected in the high value of the $\mathrm{V}_{\text {oc }}$ in the region (ii) of the curve. However, in the mixed conductor, the holes and cationic currents are interrelated and respond simultaneously to the concentration gradients created by the thermodiffusion effects. As a result, the internal field due to developing proton concentration higher at the cold side than the hot side leads to an opposite $\mathrm{h}^{+}$drift current toward the hot side. This opposite hole current limits the Soret effect of protons and eventually leads to the decline of the $\mathrm{V}_{\text {oc }}$ as seen in the region (iii) of the curve.

\subsection{Electrical output versus time}

When an external resistor is connected to the hot and cold electrodes (Figure 4a), the current versus time evolution is very different for the three types of materials: for an electronic conductor (PEDOT-Tos), the current is constantly generated because the thermodiffused holes 
reaching the cold electrode passes through the external circuit. This is the principle of thermoelectric generators. ${ }^{[26]}$ For an ionic conductor (e.g. PSSNa), the temperature gradient leads to a concentration gradient of ionic charge carriers developing slowly with time to reach a high and constant $\mathrm{V}_{\text {oc. }}$ But the ions are accumulated at the Au electrodes and cannot pass through an external circuit. Hence, the ionic thermoelectric effect can charge an electric double layer at the metal electrode. This is the principle of charging an ionic thermoelectric supercapacitors. ${ }^{[35]}$ For a mixed conductor (PEDOTPSS), the behavior is yet different. A first question is if the ionic reorganization occurs at the interface between gold electrode and PEDOT-PSS (like in the case of PSSNa) or within the bulk of the polymer film. To in part investigate this, we measure the amount of charges that flows from the cold to the hot electrodes by applying a load resistor to the two electrodes. Figure $\mathbf{4 b}$ shows the output current calculated from the voltage drop measured across a load resistor over time. The resistor value was chosen to equal the internal resistance of the device $\left(\mathrm{R}_{\mathrm{L}}=\mathrm{R}_{\mathrm{i}}=347 \Omega\right)$. When a $\Delta \mathrm{T}$ of $3.7^{\circ} \mathrm{C}$ was applied across the PEDOT-PSS film at $80 \% \mathrm{RH}$, the current rises accordingly and reaches a maximum peak value of $0.22 \mu \mathrm{A}$ as the $\Delta \mathrm{T}$ gets constant (i.e. after $\sim 100 \mathrm{~s}$ ). After reaching this peak current value, the current reduces down to $\sim 0.024 \mu \mathrm{A}$ and becomes then constant. This constant low current is attributed to a pure electronic thermoelectric effect; while the added current rise and fall occurring from $0 \mathrm{~s}$ to $\sim 250 \mathrm{~s}$ is attributed to an ionic thermoelectric effect and corresponding rearrangement of the ions within the films. The integrated charge under this bump equals an ionic charge of $\mathrm{Q}_{\mathrm{i}}=22.7$ $\mu \mathrm{C}$ rearranged in the sample. By increasing the applied temperature in 4 discrete steps finally reaching a $\Delta \mathrm{T}$ of $3.7^{\circ} \mathrm{C}$, we record a series of 4 current bumps, see Figure $4 \mathrm{c}$. The integrated charge estimated from the current versus time peak in Figure $4 \mathrm{~b}$ equals the sum of the 4 peaks recorded in the Figure 4c experiment. This then suggests that the reorganization of ionic charges in the film can occur in discrete steps and that the total amount of electrical and ionic charges involved in the corresponding reactions and polarizations is maintained. To relate the order of magnitude of the total charge involved in this thermoelectric-driven reorganization, we simply compare this total amount of charge with that stored in a typical electric double layer EDL residing along the interface between an ionic conductor 
and planar a metal surface (Heltmoltz layer, $C=100 \mu \mathrm{F} / \mathrm{cm}^{2}$ ). ${ }^{[36]}$ For a voltage that equals the maximum $\mathrm{V}_{\text {oc }}$ of $300 \mu \mathrm{V}$ of our experiment, and a capacitor surface that equals the area of the gold electrode $\left(A=1.5 \times 10^{-5} \mathrm{~m}^{2}\right)$, an EDL will then store a charge $\mathrm{Q}_{\mathrm{EDL}}=4.5 \times 10^{-3} \mu \mathrm{C}$. Hence, since $\mathrm{Q}_{\mathrm{i}}>>$ QEDL, we conclude that the ionic charges is likely reorganized throughout the entire bulk of the PEDOT-PSS film and not just along the electrode-bulk interface. This then motivates us to investigate the charge reorganization effects using a spectroscopic method that is sensitive to the chemical composition of the bulk of the PEDOT-PSS thin films.

\subsection{Spectroscopic characterization}

In order to understand the mechanism of the ionic thermoelectric effect in PEDOT-PSS, we use in situ Attenuated Total Reflectance- Fourier-transform infrared (ATR- FTIR) spectroscopy to resolve either a variation in free-carrier density or modification of the structure of the polymer chains upon exposure to a $\Delta \mathrm{T}$. A schematic view of the setup for the measurements is shown in Figure 5a. ATR-FTIR spectra of PEDOT-PSS were recorded before $(\mathrm{t}=0 \mathrm{~s})$, during $(\mathrm{t}=0-220 \mathrm{~s})$ and after $(\mathrm{t}=220-400 \mathrm{~s})$ applying $\Delta \mathrm{T}$. Spectra in the wavenumber region $1800-800 \mathrm{~cm}^{-1}$ are shown in Figure $\mathbf{5 b}$ and were recorded at the cold side of the sample. The spectrum at the $t=0$ presents the chemical composition of the pristine sample and the main vibrations are listed in Table $1 .{ }^{[37-41]}$ The broad background absorption is associated with free electronic carriers present in crystalline domains of semi-metallic character. ${ }^{[42]}$ Figure $5 c$ displays the time evolution of the total integrated intensity of the IR spectra from 1800 to $800 \mathrm{~cm}^{-1}$. After applying $\Delta \mathrm{T}$, the background intensity of the spectrum at wavenumbers greater than $1300 \mathrm{~cm}^{-1}$ gradually increases until reaching a maximum $(\mathrm{t}=80 \mathrm{~s})$. The presence of a pronounced IR background at the cold side indicates that PEDOT-PSS possesses a higher charge carrier density at the cold side as compared to the hot side. The electronic carriers are positively charged (holes), thus giving a positive sign of the $\mathrm{V}_{\text {oc }}$, which is in agreement with our measurements. After $80 \mathrm{~s}$, the intensity of the spectrum in the $1300-1800 \mathrm{~cm}^{-1}$ region decreases even when the $\Delta \mathrm{T}$ is maintained ( $\mathrm{t}=80-220 \mathrm{~s})$ and finally becomes constant after the heater is turned off 
$(\mathrm{t}=440 \mathrm{~s})$. However, the final intensity level is higher than that of the pristine film that has not been subjected to a $\Delta \mathrm{T}$. It is important to note that the time duration of the process in which the free carrier density varies under the $\Delta \mathrm{T}$ is of the same order as that of the development of the open circuit voltage in Figure 3a.

To monitor structural changes of the PEDOT-PSS film, under the exposure to the temperature gradient, bands attributed to thiophene rings (1550-1400 $\mathrm{cm}^{-1}$ ) were examined (Figure 6). After applying $\Delta \mathrm{T}$ (up to $\mathrm{t}=80 \mathrm{~s}$ ), the position of the asymmetric $\mathrm{C}_{\alpha}=\mathrm{C}_{\beta}$ stretching band shifts from 1521 to $1517 \mathrm{~cm}^{-1}$ and a new band also appears at $1471 \mathrm{~cm}^{-1}$, which correlates to the formation of $\mathrm{C}_{\beta}=\mathrm{C}_{\beta}$ bonds within the thiophene rings. At the same time, the intensity of the symmetric $\mathrm{C}_{\alpha}=\mathrm{C}_{\beta}$ stretching bands at 1492 and $1451 \mathrm{~cm}^{-1}$ decreases. All of these changes can be attributed to a structural transformation from the benzoid to quinoid structure of PEDOT, which is accompanied with the formation of positive (bi)polarons at the cold side. The density of free electronic carriers is thus correlated with the density of quinoid strucutres, i.e. bipolaron species. This further supports the hypothesis to create local a bipolaron networks that then leads to the formation of a semi-metallic electronic structure. ${ }^{[42]}$ After 80 seconds (the maximum intensity reached for free carrier density, Figure 5c), the intensities and positions of bands and peaks start to return to eventually coincide with those of the initial spectrum ( $\mathrm{t}=0 \mathrm{~s})$, which can be attributed to a compensation of the positive (bi) polarons at the cold side, and the PEDOT structure converts back to the benzoid form. Thus the PEDOT phase in the film is more reduced at the cold side.

To study the conduction mechanism in PEDOT-PSS films under the exposure to a $\Delta \mathrm{T}$, the glass substrate, with the film residing between electrodes, is placed on a pair of Peltier elements under an FTIR microscope. Figure 7 includes FTIR images and the corresponding spectra from the pristine sample, during the build-up of the $\Delta \mathrm{T}$, and then finally under the steady-state $\Delta \mathrm{T}$. The FTIR images show the integrated intensities in the $2000-800 \mathrm{~cm}^{-1}$ region. The spectra were also integrated over a line parallel to, and close to, the electrodes to provide the averaged spectra of the material closest the edges of the electrodes. The image of the pristine sample demonstrates that the structure 
of the material is similar across the sample, and the line-averaged spectra (1 and 2 in Figure 7 ) also confirm the homogeneity. After applying the $\Delta \mathrm{T}$ (of approximately $1^{\circ} \mathrm{C}$ ), the intensity increased at the cold side. Comparing the spectra at the hot and cold sides (spectra 3 and 4 in Figure 7) reveals that the intensity of the spectrum at the cold side increases at wavenumbers higher than $1300 \mathrm{~cm}^{-1}$, and supports the formation and accumulation of positively charged bipolarons at the cold side. ${ }^{\text {[43-44] }}$ Spectrum 4 also displays a weak new band appearing at $1517 \mathrm{~cm}^{-1}$, which can be related to the benzoid - quinoid reaction in PEDOT at the cold side. The FTIR image at steady-state shows that most of the bipolarons have disappeared at the cold side, due to neutralization via charge compensation of the PEDOT. The results obtained from the FTIR microscopic imaging are in good agreement with findings from the FTIR-ATR results.

\section{Discussion}

As already discussed earlier, PEDOT-PSS is a mixed conductor, wherein both electronic (holes) and ionic charge (protons) charge carriers contribute in parallel to the overall charge conduction. In order to stabilize the PEDOT-PSS emulsion in aqueous medium, an excess PSS is added; so that the PSS-to-PEDOT ratio is about 2.5. The PEDOT-PSS emulsion has a low pH, so most of the cations are protons. Only about one tenth of the sulfonate groups are balancing the positive charge on the PEDOT chains. So, the concentration ratio between holes and protons is of the order of $1 / 10$. The electronic conductivity is of the order of $30 \mathrm{~S} / \mathrm{m}$; while the ionic conductivity is about 3 S/m. ${ }^{[30,45]}$ Hence, the hole mobility is estimated to be 100 times higher than the proton mobility in those PEDOT-PSS samples.

Under the presence of a $\Delta \mathrm{T}$, holes thermodiffuse towards the cold end at first due to their higher mobility compared to the protons. $\mathrm{V}_{\text {oc }}$ developed due to this hole concentration gradient could reach about $\sim 20 \mu \mathrm{V} / \mathrm{K}$ and is found to be instantaneous with the $\Delta \mathrm{T}$ in the region (i) of the curve in Figure 3b. But, in the presence of humidity, when a $\Delta \mathrm{T}$ is applied across PEDOT-PSS films, 
in addition to holes, it is expected that there is a significant thermodiffusion of protons towards the cold end.

The IR spectroscopy analysis reveals that the migration of protons towards the cold side leads to an increased oxidation level in PEDOT at the cold side during the increase in $\mathrm{V}_{\text {oc. }}$ This is seen as a pronounced IR background due to a high free charge carrier density at the cold side, as well as a change in the bonding pattern from benzoid (hot side) to quinoid (cold side) structure. Several studies have indeed pointed out that in acidic environment, PEDOT-PSS is getting oxidized. ${ }^{[46]}$ This has been attributed to a reaction between the proton at the alpha position of the EDOT monomer located at the end of the short PEDOT chains; the positive charge of the proton is then injected in the $\pi$ electronic system through the formation of a covalent C-H bond. ${ }^{[47]}$

(1) Hot side at the beginning of the peak: PSSH $\rightarrow$ PSS $^{-}+\mathrm{H}^{+}$(thermodiffusion of proton to the cold side)

(2) Cold side at the beginning of the peak: $\mathrm{PEDOT}^{0}+\mathrm{H}^{+} \rightarrow \mathrm{PEDOT}^{+}-\mathrm{H}$ (in response to the proton thermodiffusion)

It is important to note that the high value of $\mathrm{V}_{\text {oc }}$ caused by the movement of protons creates an electric field that is leading to a reverse hole current from cold to hot side. Hence, there are internal charge reorganization that can be seen as an internal electrochemical reaction. At the hot side, there is a lack of protons, but the incoming hole current balances the negative charge of PSS- through an oxidation (3) At the cold side, the oxidized PEDOT is giving away the excess of holes to the hot side in response to the internal electric field created by the proton accumulation at the cold side.

(4) Hot side in the peak decay: PEDOT ${ }^{0}+\mathrm{PSS}^{-}+\mathrm{h}^{+} \rightarrow$ PEDOT $^{+} \mathrm{PSS}^{-}$(oxidation)

(5) Cold side in the peak decay: PEDOT $^{+}-\mathrm{H} \rightarrow \mathrm{PEDOT}^{0}-\mathrm{H}+\mathrm{h}^{+}$(reduction)

This is supported by the difference between the IR image recorded at the peak and after the peak. In this process, the IR absorption background as well as the specific vibrational modes (in Figure 5c, 
Figure 6, and Figure 7) indicate an effective electrochemical reduction of PEDOT at the cold side, and oxidation at the hot side. After the peak, the $\Delta \mathrm{T}$ is maintained but the IR camera is not able to resolve the tiny difference in oxidation level in the sample after this reorganization since the resulting $\mathrm{V}_{\text {oc }}$ is small $(20 \mu \mathrm{V})$ and the state of the sample rather close to the pristine state before the temperature was applied.

\section{Conclusions}

We demonstrate that the time dependence behavior of the $\mathrm{V}_{\text {oc }}$ is a way to distinguish between three classes of polymeric materials: electronic conductor, ionic conductor and mixed electronic-ionic conductor. The time dependence of the $\mathrm{V}_{\text {oc }}$ in a mixed ionic-electronic conductor, namely, PEDOT-PSS, shows the contribution of both charge carriers towards the Seebeck voltage. $\mathrm{V}_{\text {oc }}$ of $380 \mu \mathrm{V}$ was obtained at $100 \% \mathrm{RH}$ when a $\Delta \mathrm{T}$ of $1^{\circ} \mathrm{C}$ was applied across the film. The electrical and spectroscopic evidence show that when the conducting polymer PEDOT-PSS is under the $\Delta \mathrm{T}$ at high humidity, a thermodiffusion of protons takes place, and it is followed by an internal electrochemical reaction. This complex behavior is a result of the mixed nature of the charge carrier conduction. The polymer system behaves like a device in which the power generated by the ionic charges is consumed within the device due to the internal load $\mathrm{R}_{\mathrm{i}}$ (electronic shunt).

\section{Experimental Section}

Glass slides with two thermally evaporated gold electrodes (1 mm wide, $1 \mathrm{~mm}$ apart, $\sim 100 \mathrm{~nm}$ thick; for the $\mathrm{V}_{\text {oc }}$ measurement $1.5 \mathrm{~cm}$ long, and for the impedance measurement $53 \mathrm{~mm}$ long) were used as substrates. The polymer layers were casted onto these electrode-patterned glass substrates and dried at room temperature. The polymer layer was removed from the area outside that defined by the metal electrodes. The sample was fixed in between a pair of Peltier modules that were programmed to maintain the desired $\Delta \mathrm{T}$ across the sample. Voltage measuring probes were attached 
on the gold electrodes. $\Delta \mathrm{T}$ between the two electrodes was measured using a differential thermocouple arrangement. Both the $\mathrm{V}_{\text {ос }}$ and the $\Delta \mathrm{T}$ were measured simultaneously, but independent of each other using a Keithley Nanovoltmeter 2182A. In order to have the independent voltage and temperature measurement path, each thermocouple was kept on the $1 \mathrm{~mm} \times 1 \mathrm{~mm}$ gold electrode in line, but $1 \mathrm{~mm}$ away from the voltage measuring electrode as shown in Figure 2b. The measurements under humidity were carried out in a controlled environment chamber model 5532, electro-tech systems Inc.

The electrical conductivity of the films were measured using an impedance spectrometer (an Alpha high resolution dielectric analyzer, Novocontrol Technologies GmbH) with two-point probe. An ac voltage of $100 \mathrm{mV}$ was applied between the electrodes with a frequency sweep from $1 \mathrm{MHz}$ to $0.01 \mathrm{~Hz}$. The electrical conductivity of the samples were then calculated as $\sigma=$ $\mathrm{d} /(\mathrm{R} \times \mathrm{A})$, where $\mathrm{d}, \mathrm{R}$ and $\mathrm{A}$ are the distance between the electrodes, real part of the impedance at zero phase angle, and area of cross-section of the film, respectively.

Attenuated Total Reflectance-Fourier-transform infrared (ATR-FTIR) spectroscopy was combined with temperature gradients to study the conduction mechanism of PEDOT-PSS layers in situ. Measurements were carried out with a PIKE MIRacle ATR accessory with a diamond prism in a Vertex 70 spectrometer (Bruker) using a DLaTGS detector, and the system was continuously purged with $\mathrm{N}_{2}$. IR spectra were acquired at $4 \mathrm{~cm}^{-1}$ resolution and 32 scans between 4000 and 800 $\mathrm{cm}^{-1}$. Firstly, IR spectra were recorded from the pristine sample before switching on the heater, after that, the heater was switched on for $200 \mathrm{~s}$ at $60^{\circ} \mathrm{C}$ and each IR spectrum was recorded over a duration of 20s. IR measurements were continued even after switching off and removing the heater. The presented spectra are baseline-corrected using the same rubber.band correction for all spectra.

The FTIR imaging measurements were carried out with a Bruker Hyperion 3000 microscope using a Tensor 27 spectrometer as the light source. The microscope has $128 \times 128$ pixels focal plane array (FPA) detector, giving a lateral resolution of $2 \mu \mathrm{m}$. The system was continuously 
purged with nitrogen before and during the measurements. FTIR images were captured between 4000 and $800 \mathrm{~cm}^{-1}$ and with a $4 \mathrm{~cm}^{-1}$ spectral resolution and 64 scans. The FTIR images were captured from the sample, with a detective area of $300 \times 300 \mu \mathrm{m}^{2}$ between two planar gold electrodes, and Peltier elements were used to create $\Delta \mathrm{T}$ across the sample.

\section{Acknowledgements}

The authors acknowledge the European Research Council (ERC-starting-grant 307596), the Swedish foundation for strategic research (SSF), the Knut and Alice Wallenberg foundation (KAW), The Swedish Energy Agency and the Advanced Functional Materials Center at Linköping University. Authors would like to thank Mr. Ionut Alexandru Apolozan and Mr. Skomantas Puzinas for their help in setting up the automated Seebeck measurement setup.

[1] J. Maier, Nat. Mater. 2005, 4, 805.

[2] B. Winther-Jensen, O. Winther-Jensen, M. Forsyth, D. R. MacFarlane, Science. 2008, 321, 671.

[3] Z. Shao, S. M. Haile, Nature. 2004, 431, 170.

[4] R. W. Baker, Ind.Eng. Chem. Res. 2002, 41, 1393.

[5] H. H. Wang, S. Werth, T. Schiestel, J. Caro, Angew. Chem. 2005, 117, 7066;

Angew. Chem. Int. Ed. 2005, 44, 6906.

[6] G. Milczarek,, O. Inganäs, Science. 2012, 335, 1468.

[7] J. Rivnay, P. Leleux, A. Hama, M. Ramuz, M. Huerta, G. G. Malliaras, R. S. Owens, Sci. Rep. 2015, 5, 11613,

[8] S-H. Park, H-I. Yoo, Phys. Chem. Chem. Phys. 2009, 11, 391.

[9] I. Riess, Solid State Ionics. 2003, 157,1. 
[10] A.Malti, J. Edberg, H. Granberg, Z. U. Khan, J. W. Andreasen, X. Liu, D. Zhao, H. Zhang, Y.Yao, J. W. Brill, I. Engquist, M. Fahlman, L. Wågberg, X. Crispin, M. Berggren, Adv. Sci. 2016, 3, 1500305.

[11] Y. Lin, S. Fang, D. Su, K. S. Brinkman, F. Chen, Nat. Commun. 2015, 6, 6824.

[12] H. Liu, X. Shi, F. Xu, L. Zhang, W. Zhang, L. Chen, Q. Li, C. Uher, T. Day, G. J. Snyder, Nat. Mater. 2012, 11, 422.

[13] D. R. Brown, T. Day, K. A. Borup, S. Christensen, B. B. Iversen, G. J. Snyder, APL Mater. 2013, 1, 052107.

[14] H. Liu, X. Shi, M. Kirkham, H. Wang, Q. Li, C. Uher, W. Zhang,L. Chen, Mater. Lett. 2013, 93, 121.

[15] T. W.Day, K. A. Borup, T. Zhang, F. Drymiotis, D. R. Brown, X. Shi, L. Chen, B. B. Iversen, G. J. Snyder, Mater. Renew. Sustain. Energy. 2014, 3, 26.

[16] F. Gascoin, A. Maignan, Chem. Mater. 2011, 23, 2510.

[17] M. Ferhat, J. Nagao, J. Appl. Phys. 2000, 88, 813.

[18] T. Day, F. Drymiotis, T. Zhang, D. Rhodes, X. Shi, L. Chen, G. J. Snyder, J. Mater. Chem. C. 2012, 1, 7568.

[19] W. Koch, H. Rickert, G. Schlechtriemen, Solid State Ionics. 1983, 9-10, 1197.

[20] C. Korte, J. Janek, H. Timm, Solid State Ionics 1997, 101-103, 465.

[21] M. A. Korzhuev, A. V. Laptev, Fiz. Tverd. Tela (Leningrad). 1987, 29, 2646. (Sov. Phys. Solid State. $1987,29,1524$.

[22] S-H. Park, H-I. Yoo, Phys. Chem. Chem. Phys. 2009, 11, 391.

[23] P. Andersson, D. Nilsson, P. O. Svensson, M. Chen, A. Malmström, T. Remonen, T. Kugler, M. Berggren, Adv. Mater. 2002, 14, 1460.

[24] Q. Pei, G. Zuccarello, M. Ahlskog, O. Inganäs, Polymer. 1994, 35, 1347.

[25] T. Park, C. Park, B. Kim, H. Shin, E. Kim, Energ. Environ. Sci. 2013, 6, 788. 
[26] O. Bubnova, Z. U. Khan, A. Malti, S. Braun, M. Fahlman, M. Berggren, X. Crispin, Nat. Mater. 2011, 10, 429.

[27] S. H. Lee, H. Park, S. Kim, W. Son, I. W. Cheong, J. H. Kim, J. Mater.

Chem. A 2014, 2, 7288.

[28] G. H. Kim , L. Shao , K. Zhang , K. P. Pipe , Nat. Mater. 2013, 12, 719.

[29] Q.S. Wei, M. Mukaida, K. Kirihara, Y. Naitoh, T. Ishida, Appl. Phys. Express. 2014, 7, 031601.

[30] H. Wang, U. Ail, R. Gabrielsson, M. Berggren, X. Crispin, Adv. Energy Mater. 2015, 5, 1500044.

[31]. K. E. Aasmundtveit , E. J. Samuelsen , L. A. A. Pettersson ,O. Inganäs , T. Johansson , R. Feidenhans, Synth. Methods. 1999, 101, 561.

[32] O. Larsson, E. Said, M. Berggren, X. Crispin, Adv. Funct. Mater. 2009, 19, 3334

[33] X. Crispin, M. Berggren, H. Wang, (Acreo Swedish ICT AB164 40 Kista (SE)) EP 2658003 A1, 2013.

[34] H. J. V. Tyrrell, D. A. Taylor, C. M. Williams, Nature. 1956, 177, 668.

[35] D. Zhao, H. Wang, Z. U. Khan, J. C. Chen, R. Gabrielsson, M. P. Jonsson, M. Berggren, X. Crispin, Energy Environ. Sci. 2016, DOI: 10.1039/c6ee00121a.

[36] M. E. Orazem, B. Tribollet. Electrochemical Impedance Spectroscopy, Chapter 5 Electrochemistry. John Wiley \& Sons, 2008.

[37] S. Xiong, L. Zhang, X. Lu, Polym. Bull. 2013, 70, 237,

[38] F. Ely, A. Matsumoto, B. Zoetebier, V. S. Peressinotto, M. K.Hirata, D. A. de Sousa, R. Maciel, Org. Electron. 2014, 15, 1062.

[39]C. Kvarnström, H. Neugebauer, S. Blomquist, H.J. Ahonen, J. Kankare, A. Ivaska Electrochim. Acta. 1999, 44, 2739.

[40] L. Sheng, T. Higashihara, S. Nakazawa, M. Ueda Polym. Chem. 2012, 3, 3289. 
[41] S. Garreau, G. Louarn, J. P. Buisson, G. Froyer, and S. Lefrant, Macromolecules. 1999, 32, 6807.

[42] O. Bubnova, Z.U. Khan, H. Wang, S. Braun, D. R. Evans, M. Fabretto, P. H-Talemi, D. Dagnelund, J-B. Arlin, Y. H. Geerts, S. Desbief, D. W. Breiby, J. W. Andreasen, R. Lazzaroni, W. M. Chen, I. Zozoulenko, M. Fahlman, P. J. Murphy, M. Berggren, X. Crispin, Nat. Mater. 2014, 13, 190.

[43] Z. Q. Li, G. M. Wang, N. Sai, D. Moses, M. C. Martin, M. Di Ventra, A. J. Heeger, D. N. Basov, Nano Lett. 2006, 6, 224.

[44] J. Hwang and D. B. Tanner, Phys. Rev. B. 2003, 67, 115205.

[45] Mark Lefebvre Zhigang Qi Danesh Rana Peter G. Pickup Chem. Mater. 1999, 11, 262.

[46] T.-C. Tsai, H.-C. Chang, C.-H. Chen, Y.-C. Huang, W.-T. Whang, Org. Electron. 2014, 15, 641.

[47]. Z.U. Khan, O. Bubnova, M. J. Jafari, R. Brooke, X. Liu, R. Gabrielsson, T.Ederth, D. R. Evans, J. W. Andreasen, M. Fahlman, X. Crispin, J. Mater. Chem. C. 2015, 3, 10616. 
Table 1: Positions of IR absorption bands, and the corresponding vibrational modes.

\begin{tabular}{|c|c|c|}
\hline $\begin{array}{l}\text { Band position } \\
\qquad\left(\mathrm{cm}^{-1}\right)\end{array}$ & Band assignment & References \\
\hline $1636,1601,1589$ & $\mathrm{C}=\mathrm{C}$ stretching, phenyl side group of PSS & [37] \\
\hline 1521 & Asymmetric $\mathrm{C}_{\alpha}=\mathrm{C}_{\beta}$ stretching, thiophene ring & {$[37,38]$} \\
\hline 1492, & Symmetric $\mathrm{C}_{\alpha}=\mathrm{C}_{\beta}$ stretching, thiophene ring & [38] \\
\hline 1451 & Symmetric $\mathrm{C}_{\alpha}=\mathrm{C}_{\beta}(-\mathrm{O})$ stretching, thiophene ring & [38] \\
\hline 1369,1356 & C-C stretching, thiophene ring & [38] \\
\hline 1294 & $\begin{array}{c}\text { Asymmetric } \mathrm{S}=\mathrm{O} \text { stretching, } \mathrm{PSS} ; \mathrm{C}_{\alpha}-\mathrm{C}_{\alpha} \text {, inter-ring } \\
\text { stretching, PEDOT backbone }\end{array}$ & {$[37,38,40]$} \\
\hline $\begin{array}{l}1190 \text { (shoulder), } \\
1179\end{array}$ & Symmetric $\mathrm{S}=\mathrm{O}$ stretching, PSS & {$[37,40]$} \\
\hline $1127,1068,1029$ & C-O-C stretching and ring vibration, ethylenedioxy ring & [37-39] \\
\hline 1006 & S-phenyl stretching, PSS & [38] \\
\hline 964,834 & C-S stretching, thiophene ring & {$[37,39]$} \\
\hline 907 & C-H deformation, ethylenedioxy ring & [41] \\
\hline
\end{tabular}




PEDOT-Tos

Figure 1. Structure and charge carriers in the PEDOT-Tos, PEDOT-PSS and PSSNa 


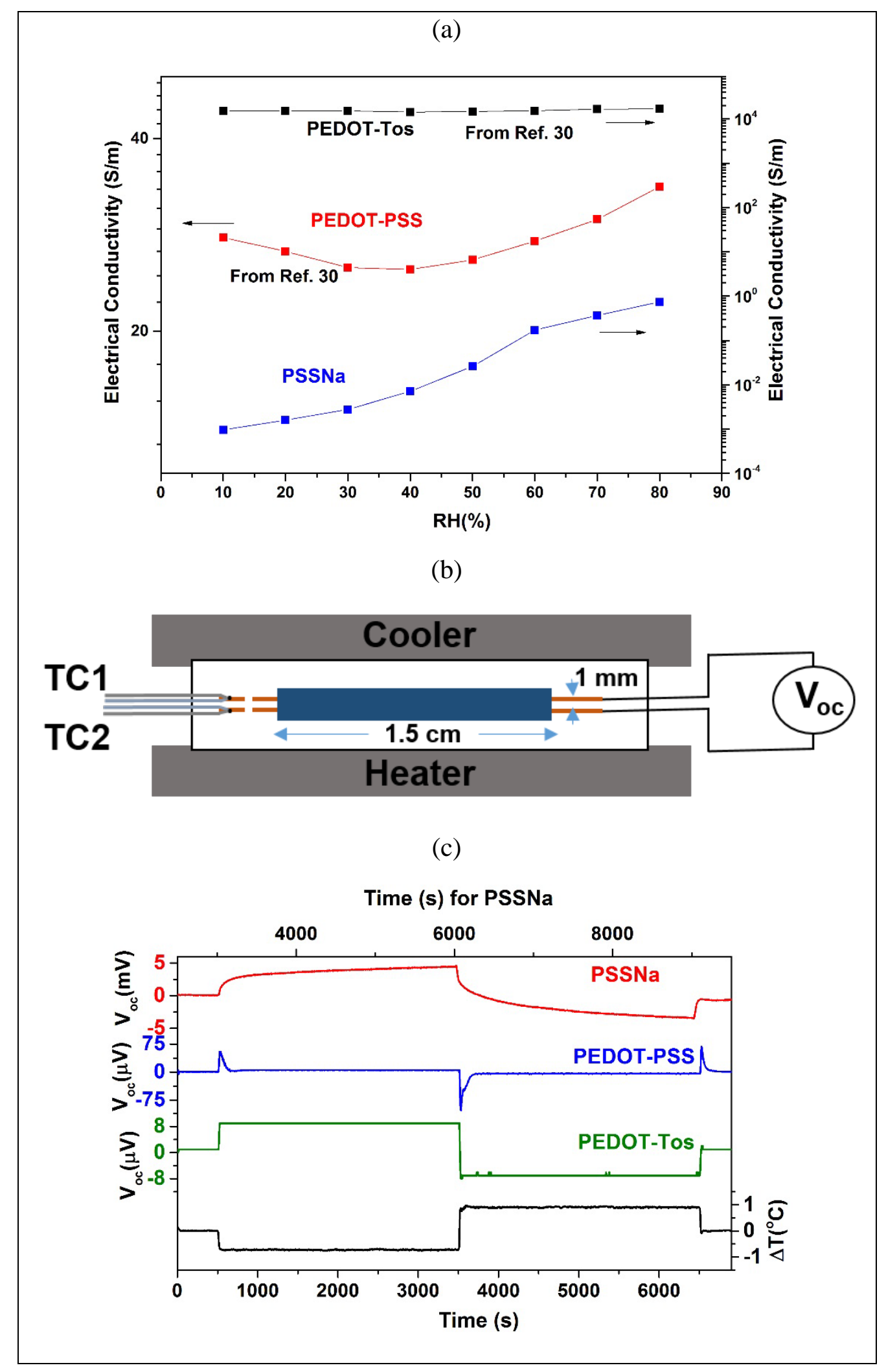

Figure 2. (a) Electrical conductivity as a function of humidity for PEDOT-Tos, PEDOT-PSS, and PSSNa. (b) Schematic of the device for measuring the open circuit voltage $V_{\text {oc }}$ with time (c) Open circuit voltage vs. time for PEDOT-Tos, PEDOT-PSS, and PSSNa, at $80 \% \mathrm{RH}$ with $\Delta \mathrm{T}=1$ ${ }^{\circ} \mathrm{C}$. 


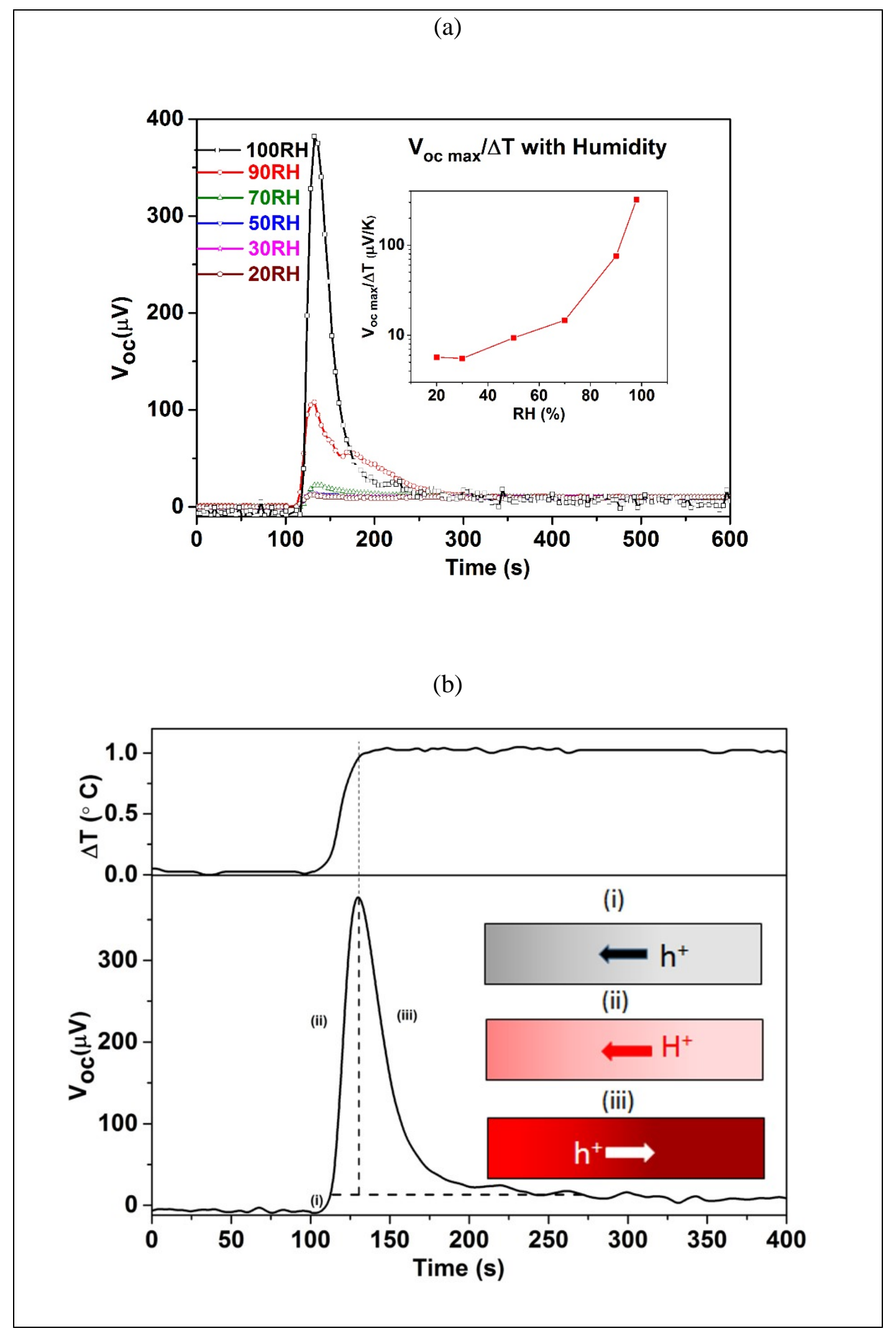

Figure 3. (a) Open circuit voltage as a function of humidity for PEDOT-PSS. (b) Different regions of the $\mathrm{V}_{\mathrm{oc}}$ - time curve under a $\Delta \mathrm{T}$ and the corresponding mechanism. 
(a)

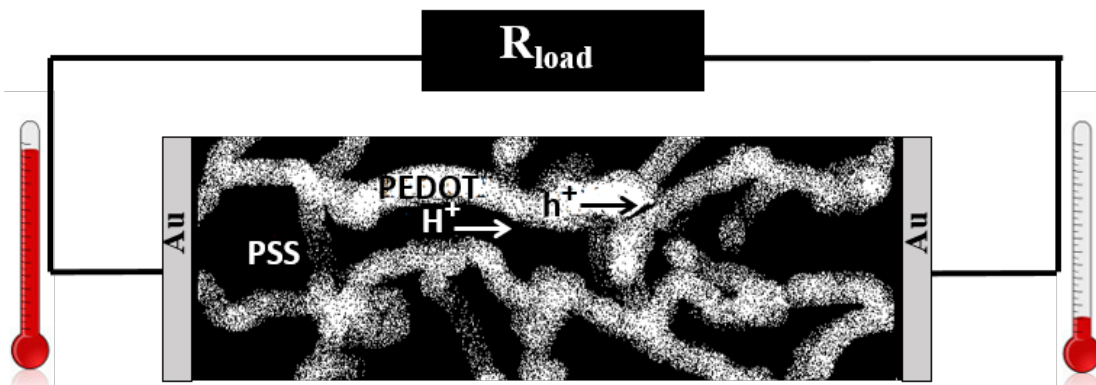

(b)

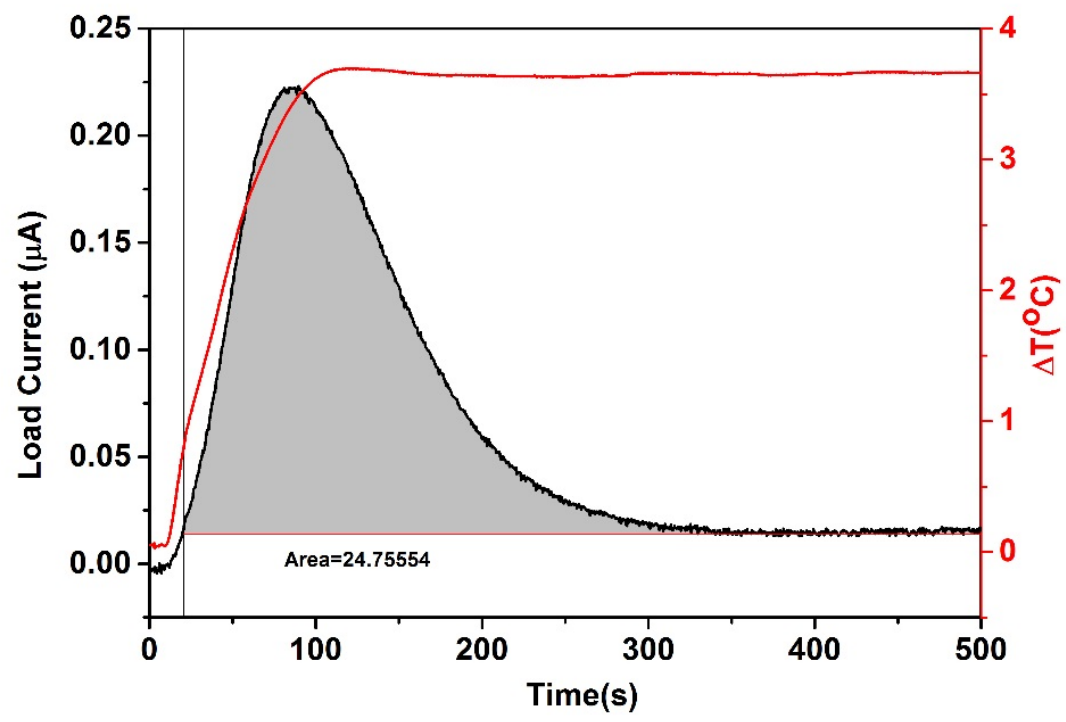

(c)

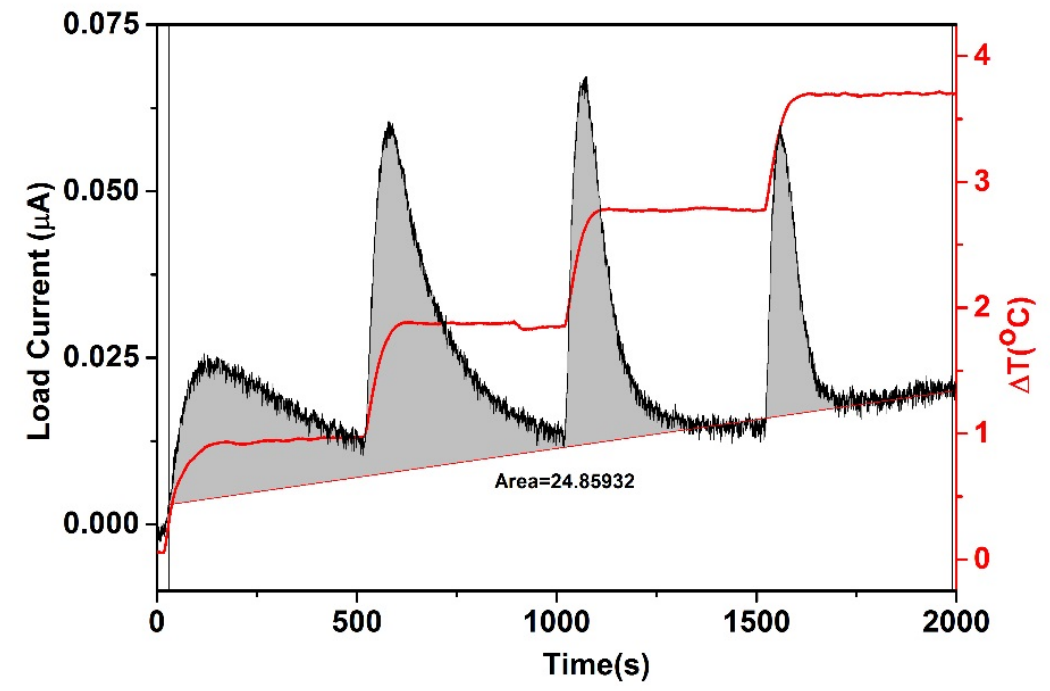

Figure 4. (a) Schematic of the PEDOT-PSS film morphology along with the setup used for the measurement of output voltage across a load. The current calculated from the voltage drop across the 
load resistor with resistance equal to the internal resistance of the device by applying a $\Delta \mathrm{T}$ in (b) a single step and with (c) multiple steps, respectively.

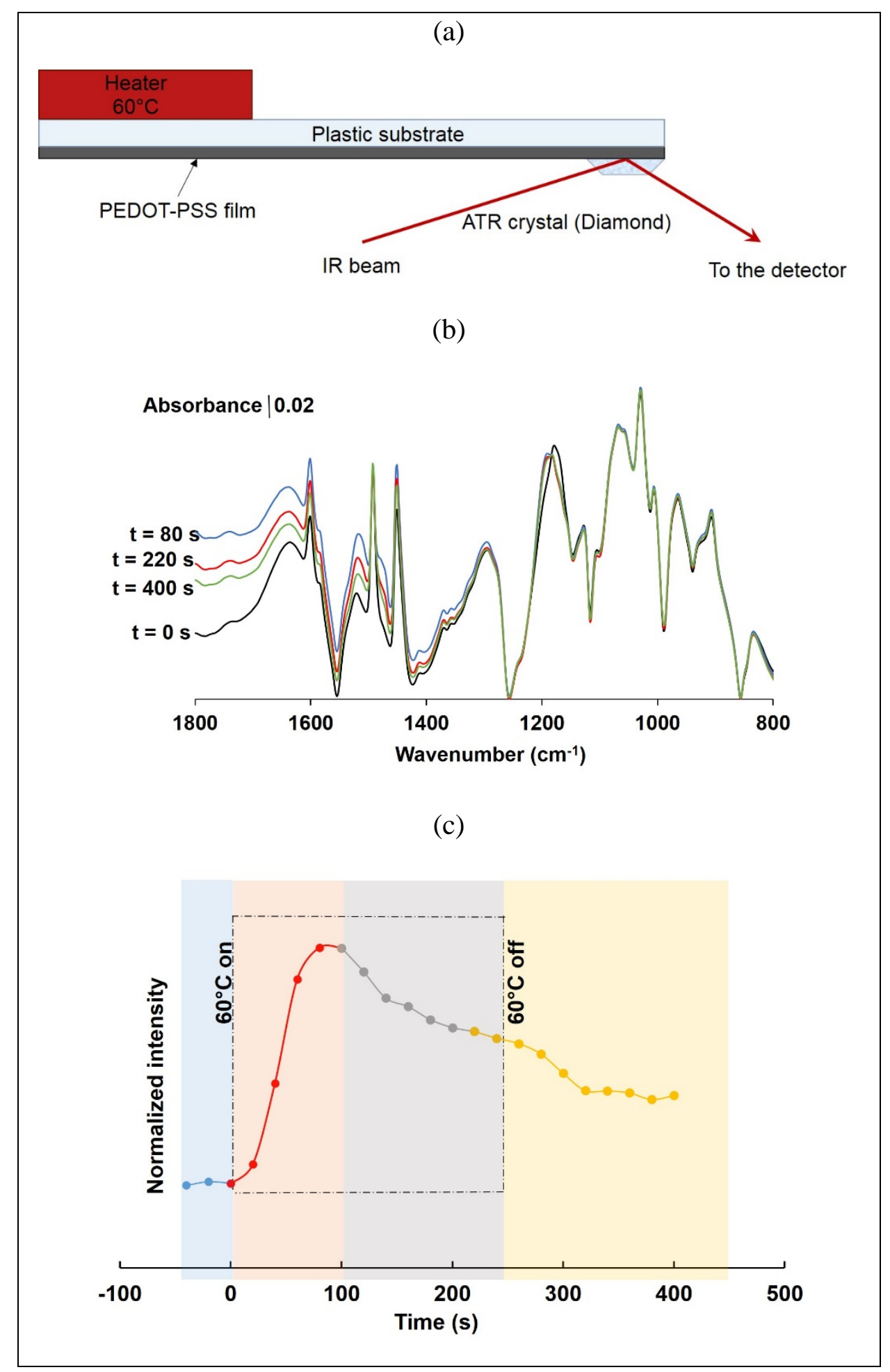

Figure 5. (a) Schematic representation of the ATR-FTIR setup for in-situ spectral measurements. (b) ATR-FTIR spectra of PEDOT-PSS recorded as a function of time under temperature a gradient. The spectrum at $\mathrm{t}=0 \mathrm{~s}$ is for pristine sample; $\mathrm{t}=80$ and $220 \mathrm{~s}$ under $\Delta \mathrm{T} ; \mathrm{t}=400 \mathrm{~s}$ at the cold side after switching off the heater. (c) Variation of the normalized integrated area of spectra from 1800 
to $800 \mathrm{~cm}^{-1}$, indicative of a change in the density of free electronic carriers with time, under the temperature gradient

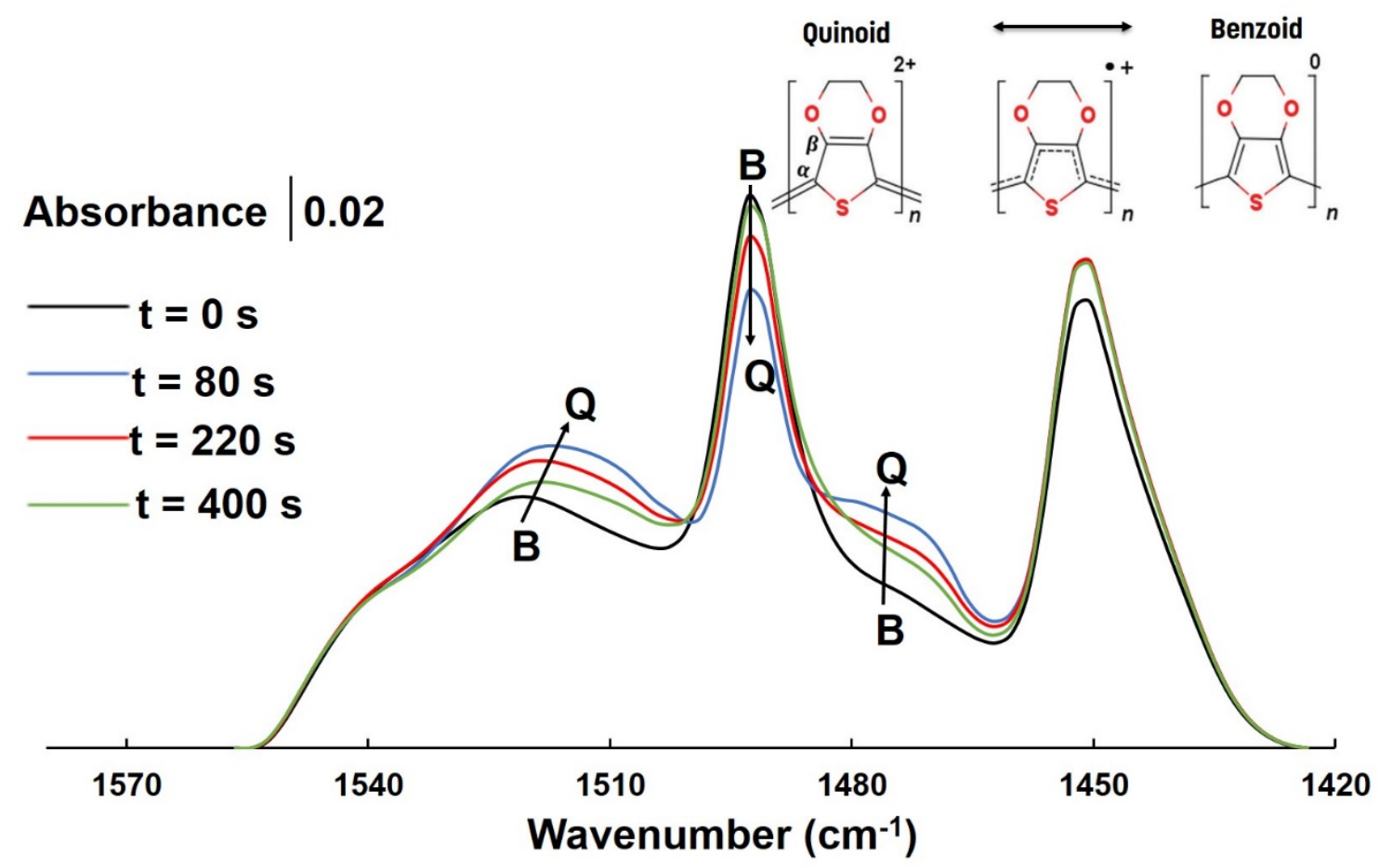

Figure 6. FTIR spectra showing the variation of the bands corresponding to the thiophene with time under the applied $\Delta \mathrm{T}$. The inset shows the chemical structure of the monomer units when carrying no charge (benzoid) or positive charge (radical cation or dication) with a quinoid character. 

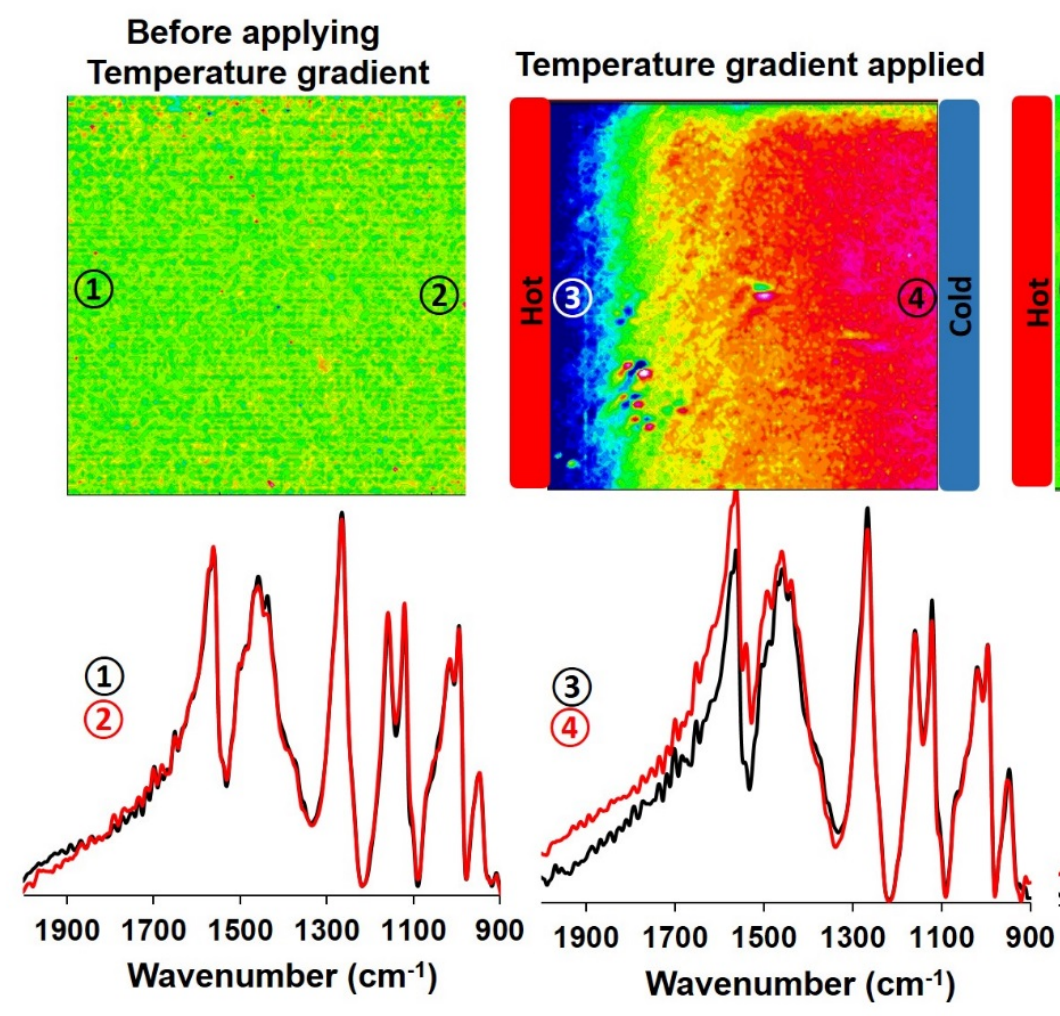

Stable state under

Temperature gradient

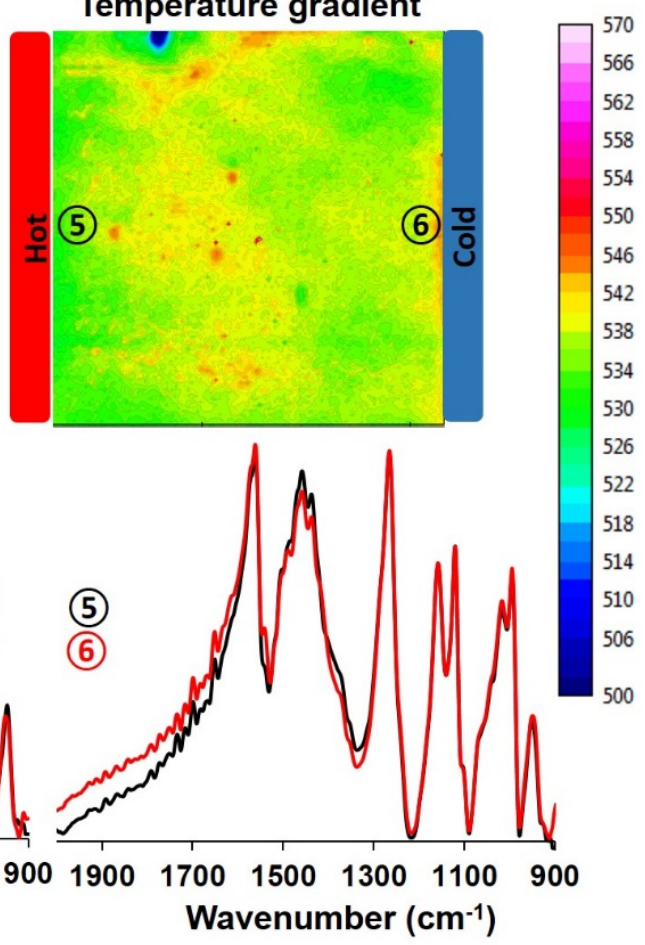

Figure 7. FTIR microscopic images of a pristine PEDOT-PSS film (left), a after the application of a $\Delta \mathrm{T}$ (middle), and under steady-state conditions under an applied $\Delta \mathrm{T}$ (right), revealing the distributions of bipolarons in the film. The line-averaged FTIR spectra were obtained at the indicated positions (and integrated along lines parallel to the electrodes at the cold and hot edges). The images show an area of 300 by $300 \mu \mathrm{m}^{2}$ 